\title{
Chemisches Zentralblatt
}

\section{Neue Bücher}

$\mathrm{E}_{1}$. Allgemeine Biologie und Biochemie

Hans yon Euler, Beobachtungen und Betrachtungen über die am Tumor-Stoffwechsel beteiligten Wirkstoffe. Leipzig: J. A. Barth. 1961. (29 S.) $4^{0}=$ Nova Acta Leopoldina. N. F. Bd. 24. Nr. 149.

一, Cancer chemotherapy. Springfield (Illinois): Thomas; Oxford: Blackwell Scientific. 1961. (XII + $253 \mathrm{~s}$. m. Abb., Tab. u. Diagr.) = American lecture series no. $439.84 \mathrm{~s}$.

-, Membrane transport and metabolism. Proceedings of a symposium held in Prague 1960. Prag: Verl. der Tschechoslowakischen Akademie der Wissenschaften. 1961. (612 S. m. 183 Abb.) $8^{\circ}$. Kcs 68.50.

$\mathbf{E}_{3}$. Mikrobiologie. Bakteriologie. Immunologie

Wilhelm Schwartz und Adelheid Schwartz, Grundriß der allgemeinen Mikrobiologie. Teil 1. Zweite verb. $u$. erg. Aufl. Berlin: de Gruyter. 1960. (147 S. m. 26 Abb.) $8^{\circ}=$ Sammlung Göschen. Bd. 1155. DM 3,60.

$\mathbf{E}_{\mathbf{4}}$. Pflanzenchemie. Pflanzenphysiologie. Pflanzenpathologie

Leo Brauner, Das kleine pflanzenphysiologische Praktikum. Anleitung zu bodenkundlichen und pflanzenphysiologischen Versuchen für Hoch-, Ober- und Fachschulen. 6. Aufl. Jena: G. Fischer. 1961. (VIII + 278 S. m. 150 Abb.) $8^{\circ}$. DM 26,60 .

$\mathrm{E}_{5}$. Tierchemie. Tierphysiologie. Tierpathologie

Brian Anthony Fox and Allan Gillies Cameron, A chemical approach to food and nutrition. London: University of London P. 1961. (326 S. m. Abb., Tab. u. Diagr.) $30 \mathrm{~s}$.

Vernon M. Ingram, Hemoglobin and its abnormalities. Springfield (Illinois): Thomas; Oxford: Blackwell Scientific. 1961. (XIV + $153 \mathrm{~S}$. m. Abb., Tab. u. Diagr.) = American lectures series no. 416. $60 \mathrm{~s}$.

Johannes Jochlms, Hrsg., Die Bedeutung der Nahrungsfette in der Pädiatrie. Vorträge und Diskussionen auf dem Symposion am 29. September 1960 in Bad Wildungen. Stuttgart: Enke. 1961. (131 S. m. $48 \mathrm{Abb}$. u. $31 \mathrm{Tab}$ ) $8^{\circ}$. DM 27, -

Joachim Kahnau, Die Rolle der Fette in unserer Ernährung. Die natriumarme Kost und ihre Gestaltung im besonderen durch diätetische Lebensmittel. Von Walter Becker. Vortrăge. Frankfurt/Main: Verband d. Diätet. Lebensmittelindustrie. 1960. (31 S.) $8^{\circ}=$ Schriftenreihe des Verbandes der Diätet. Lebensmittelindustrie. Heft 9 . Nicht im Buchhandel.

Joseph Schumacher, Index zum Diabetes mellitus. Eine internationale Bibliographie. München, Berlin: Urban \& Schwarzenberg. 1961. (XLVIII + 877 S.) $8^{\circ}$. DM 286, 一. 

$\mathbf{E}_{6}$. Pharmakologie. Therapie. Toxikologie. Hygiene

Z. M. Bacu, Pharmacodynamie biochemique. 2e édit. Paris: Masson. 1961. (XVI + 1228 S. m. Abb.) $8^{00}$ $140 \mathrm{NF}$.

Friedrich Doenecke, Hrsg., Therapie der Infektionskrankheiten. Diagnostik und Behandlung von Leberkrankheiten. Medikamentöse und diătetische Therapie des Diabetes mellitus. Vorträge. München: Werk-Verl. 1961. (197 S. m. Abb.) 8 $8^{0}$. DM. 15,35.

Peter Gustav Hesse, Hrsg., Beiträge zur modernen Therapie. 3. Vorträge und Diskussionsbemerkungen der 6. Weimarer Therapietagung 1960. Jena: G. Fischer. 1961. (XII +481 S. m. 227 Abb. u. 29 Tab.) $8^{\circ}$. DM 54,40 .

Richard Jeffery Jones and Louis Cohen, Chemistry and therapy of chronic cardiovascular disease. Spring field (Illinois): Thomas; Oxford: Blackwell Scientific. 1961. (XVI + $200 \mathrm{~S}$. m. Tab. u. Diagr.) $=$ American lecture series no. $438.60 \mathrm{~s}$.

Fernand Mercler et René Clogne, Manuel de pharmacologie. 8 e edit. Paris: Poinat. 1960. (471 S.)

Manfred Meyer, Hrsg., Berliner Symposion über Anaesthesieprobleme des offenen Thorax. Vom 28.-30. Oktober 1959 in der Deutschen Akademie der Wissenschaften zu Berlin. Berlin: Akademie-Verl. 1961. (VIII + 154 S. m. $82 \mathrm{Abb}$. u. 13 Tab.) $4^{\circ}=$ Abhandlungen der Deutschen A kademie der Wissenschaften zu Berlin. Klasse für Medizin. Jahrgang 1961, Nr, 1. DM 18,一.

H. VIII. Metallurgie. Korrosion

Lidiya Iosiforna Sokol'skaya, Gases in light metals. Oxford, London: Pergamon. 1961. (VIII + 142 S. m. Abb., Tab. u. Diagr.) $60 \mathrm{~s}$.

W. I. Karmasin, Moderne Methoden der magnetischen Anreicherung von Eisenerzen. Moskau: Gossgortechisdat. 1961. (20 Bogen.) 1 Rbl. 10 Kop. [Russ.]

XV. Gärungsindustrie

Gunthor Müller, Die Hefen. Wittenberg: Ziemsen. 1961. (92 S. m.64 Abb.) $8^{\circ}=$ Die neue Brehm-Bücherei. 279. DM 5,20 .

\section{Ausgewertete Bibliographie-Fachzeitschriften}

\begin{tabular}{ll|l} 
Belgien & $\begin{array}{l}\text { Bibliographie de Belgique - Belgi- } \\
\text { sche Bibliographie }\end{array}$ & England \\
CSSR & $\begin{array}{l}\text { Bulletin - Die Neuesten Forschungs- } \\
\text { ergebnisse der Tschechoslowakischen } \\
\text { Wissenschaft } \\
\text { Nové Knihy (Neue Bücher) }\end{array}$ & \\
& $\begin{array}{l}\text { Novinky (Neuheiten) } \\
\text { Dänemark }\end{array}$ & Frankreich \\
Deutschland & $\begin{array}{l}\text { Börsenblatt für den Deutschen Buch- } \\
\text { handel - Frankfurter Ausgabe }\end{array}$ & Italien \\
& $\begin{array}{l}\text { Börsenblatt für den Deutschen Buch- } \\
\text { handel - Leipziger Ausgabe }\end{array}$ & Oapan \\
& $\begin{array}{l}\text { Deutsche Bibliographie. Wöchent- } \\
\text { liches Verzeichnis. Ausgabe II. } \\
\text { [Frankfurt/Main] }\end{array}$ & Schweiz \\
& $\begin{array}{l}\text { Deutsche Nationalbibliographie. } \\
\text { Reihe A: Neuerscheinungen des }\end{array}$ & \\
& Buchhandels [Leipzig]
\end{tabular}

British Books (Incorporating the Publishers Circular \& Booksellers Record)

British National Bibliography

Bibliographie de la France

Nieuwsblad voor de Boekhandel

Bibliografia Nazionale Italiana

Nohon Shūho (Current Publications)

Osterreichische Bibliographie

Schweizer Buch. Serie A und Serie B

Bücherverzeichnis des Jahres

(Кннжная летопись)

Neue Bücher (Новые книги)

Publishers' Weekly 